\title{
OPEN Lactobacillus acidophilus LA5 improves saturated fat-induced obesity mouse model through the enhanced intestinal Akkermansia muciniphila
}

Thunnicha Ondee ${ }^{1}$, Krit Pongpirul ${ }^{1,2,3 凶}$, Peerapat Visitchanakun ${ }^{4}$, Wilasinee Saisorn ${ }^{4}$, Suthicha Kanacharoen ${ }^{5}$, Lampet Wongsaroj ${ }^{6,7}$, Chitrasak Kullapanich ${ }^{6,7}$, Natharin Ngamwongsatit ${ }^{8}$, Sarn Settachaimongkon ${ }^{9,10}$, Naraporn Somboonna ${ }^{6,7}$ \& Asada Leelahavanichkul ${ }^{4,11 \bowtie}$

Obesity, a major healthcare problem worldwide, induces metabolic endotoxemia through the gut translocation of lipopolysaccharides (LPS), a major cell wall component of Gram-negative bacteria, causing a chronic inflammatory state. A combination of several probiotics including Lactobacillus acidophilus 5 (LA5), a potent lactic acid-producing bacterium, has previously been shown to attenuate obesity. However, data on the correlation between a single administration of LA5 versus microbiota alteration might be helpful for the probiotic adjustment. LA5 was administered daily together with a high-fat diet (HFD) for 8 weeks in mice. Furthermore, the condition media of LA5 was also tested in a hepatocyte cell-line (HepG2 cells). Accordingly, LA5 attenuated obesity in mice as demonstrated by weight reduction, regional fat accumulation, lipidemia, liver injury (liver weight, lipid compositions, and liver enzyme), gut permeability defect, endotoxemia, and serum cytokines. Unsurprisingly, LA5 improved these parameters and acidified fecal $\mathrm{pH}$ leads to the attenuation of fecal dysbiosis. The fecal microbiome analysis in obese mice with or without LA5 indicated; (i) decreased Bacteroidetes (Gram-negative anaerobes that predominate in non-healthy conditions), (ii) reduced total fecal Gramnegative bacterial burdens (the sources of gut LPS), (iii) enhanced Firmicutes (Gram-positive bacteria with potential benefits) and (iv) increased Verrucomycobia, especially Akkermansia muciniphila, a bacterium with the anti-obesity property. With LA5 administration, $A$. muciniphila in the colon were more than 2,000 folds higher than the regular diet mice as determined by $16 \mathrm{~S}$ rRNA. Besides, LA5 produced anti-inflammatory molecules with a similar molecular weight to LPS that reduced cytokine production in LPS-activated HepG2 cells. In conclusion, LA5 attenuated obesity through (i) gut dysbiosis attenuation, partly through the promotion of $A$. muciniphila (probiotics with the difficulty in preparation processes), (ii) reduced endotoxemia, and (iii) possibly decreased liver injury by producing the anti-inflammatory molecules.

${ }^{1}$ Department of Preventive and Social Medicine, Faculty of Medicine, Chulalongkorn University, Bangkok 10330, Thailand. ${ }^{2}$ Department of International Health, Johns Hopkins Bloomberg School of Public Health, Baltimore, MD, USA. ${ }^{3}$ Bumrungrad International Hospital, Bangkok 10110, Thailand. ${ }^{4}$ Department of Microbiology, Faculty of Medicine, Chulalongkorn University, Bangkok 10330, Thailand. ${ }^{5}$ Department of Biology, Krieger School of Arts and Sciences, Johns Hopkins University, Baltimore, MD, USA. ${ }^{6}$ Department of Microbiology, Faculty of Science, Chulalongkorn University, Bangkok 10330, Thailand. ${ }^{7}$ Microbiome Research Unit for Probiotics in Food and Cosmetics, Chulalongkorn University, Bangkok 10330, Thailand. ${ }^{8}$ Department of Clinical Sciences and Public Health, Faculty of Veterinary Science, Mahidol University, Nakhon Pathom 73170, Thailand. ${ }^{9}$ Department of Food Technology, Faculty of Science, Chulalongkorn University, Bangkok 10330, Thailand. ${ }^{10}$ Emerging Processes for Food Functionality Design Research Unit, Chulalongkorn University, Bangkok 10330, Thailand. ${ }^{11}$ Translational Research in Inflammation and Immunology Research Unit (TRIRU), Department of Microbiology, Chulalongkorn University, Bangkok 10330, Thailand. ${ }^{\varpi}$ email: doctorkrit@gmail.com; aleelahavanit@gmail.com 
Obesity, a major healthcare problem worldwide ${ }^{1}$, is associated with diabetes, dyslipidemia, and cardiovascular disease that leads to several major complications in critically-ill patients ${ }^{1}$. Interestingly, obesity-induced chronic inflammation leads to atherosclerosis which is a key vascular complication in obesity ${ }^{2}$. Despite the prevalence of obesity-induced inflammation, the pathogenesis of this condition is still uncertain but is possibly a combination of several mechanisms including (i) immune responses toward adipocyte injury from hypertrophic adipocyte hypoxia and adipocyte apoptosis ${ }^{3,4}$, (ii) reduction of adiponectin together with leptin elevation, (iii) metabolic dysregulation and mitochondria dysfunction and (iv) gut permeability defect (gut-leakage) induced endotoxemia (metabolic endotoxemia) ${ }^{5}$. Among these, the interaction against endotoxins may lead to the most potent response because immune activation by the organism's pathogen-associated molecular patterns (PAMPs) is naturally more severe than the response towards the host cell's damage-associated molecular patterns (DAMPs) ${ }^{6}$. Endotoxin (lipopolysaccharide; LPS) has a molecular weight of $10-100 \mathrm{kDa}$ and is found in the cell walls of Gram-negative bacteria, which are the most abundant organisms of gut microbiota ${ }^{7}$. Under normal conditions, molecules weighing over $600 \mathrm{Da}$ are unable to pass through the intestinal tight junction barrier. However, obesity and a high-fat diet (HFD) cause gut dysbiosis ${ }^{8}$ (an alteration of organisms in the intestine ${ }^{9}$ ), enhancing gut-mucosal injury enough to allow the direct translocation of high molecular weight (MW) molecules, such as LPS, into the liver and circulatory system ${ }^{10,11}$. Indeed, non-alcoholic fatty liver disease (NAFLD), also known as obesity-induced steatohepatitis, is a key complication in obesity that is worsened by LPS ${ }^{12}$.

However, gut-leakage in obesity ${ }^{10}$ is attenuated by host-beneficial probiotics ${ }^{13-15}$, partly through the reduction of endotoxemia and systemic inflammation. Additionally, some probiotics produce high MW anti-inflammatory substances ${ }^{16-20}$. Thus, beneficial molecules secreted by probiotics may also be delivered alongside harmful PAMPs into the circulatory system during gut leakage. Although (i) Lactobacillus spp. attenuates gut dysbiosis in several animal models ${ }^{20-25}$ and (ii) Lactobacillus acidophilus (LA), in combination with other probiotics, attenuates hyperlipidemia, overweight and obesity-induced hepatitis (steatohepatitis) ${ }^{12,26-29}$, the effect of LA alone upon obesity and steatohepatitis is still unclear. As such, it is easier to determine the impact of microbiota alteration after the administration of probiotics in a single strain than a combination of several strains. The promotion of beneficial gut microorganisms in response to probiotics may be as important as the advantageous effect of probiotics themselves ${ }^{30}$. Fascinatingly, commercial probiotics can be administered to target the growth of difficult-to-culture bacteria in the gut. For instance, strictly anaerobic Akkermansia muciniphila can be promoted by commonlyavailable Lactobacilli ${ }^{31}$. Hence, Lactobacillus acidophilus LA5 (LA5) was used as a single strain probiotic in a mouse model with saturated fat induced-obesity and was also tested in the hepatocyte cell-line experiments.

\begin{abstract}
Materials and methods
Animals and animal model. The animal care and use protocol was approved by the Institutional Animal Care and Use Committee of the Faculty of Medicine, Chulalongkorn University, Bangkok, Thailand (SST 025/2563) in compliance with US National Institutes of Health standards. This study was carried out in compliance with the ARRIVE guidelines. The 8-week-old male C57BL/6 mice were purchased from the National Laboratory Animal Center, Nakhorn Pathom, Thailand. Mice with a regular diet with standard laboratory chow containing fat $(4.5 \% \mathrm{w} / \mathrm{w})$ with energy content calculated at $3.04 \mathrm{kcal} / \mathrm{g}$ (Mouse Feed Food No.082, C.P. Company, Bangkok, Thailand) or high-fat diet (HFD) containing fat, mostly from lard $(60 \% \mathrm{w} / \mathrm{w})$, with energy content calculated at $8.64 \mathrm{kcal} / \mathrm{g}^{32}$ were orally administered Lactobacillus acidophilus LA5 (LA5) (Chr. Hansen, Hørsholm, Denmark) daily at $1 \times 10^{8}$ colonies forming unit (CFU) in $0.5 \mathrm{ml}$ phosphate buffer solution (PBS) or PBS alone for 2 months before sacrifice with cardiac puncture under isoflurane anesthesia. Livers were snap-frozen in liquid nitrogen and kept at $-80^{\circ} \mathrm{C}$ before use. Feces from all parts of the colon were combined and collected for microbiome analysis. Besides, fecal samples with the intestinal tissue, including the duodenum (distal to the pyloric sphincter), jejunum (central section of the small intestine), ileum (proximal to the cecum), and colon (distal to the cecum), from obese mice with or without LA5 were collected to measure Lactobacillus spp. and Akkermansia spp. content. Organs were weighed, homogenized, and centrifuged to separate the supernatant for bacterial DNA detection.
\end{abstract}

Gut leakage measurement. Gut permeability was determined by fluorescein isothiocyanate dextran (FITC-dextran) assay, endotoxemia, and immunofluorescent detection of a tight junction protein (zonaoccludens-1; ZO-1) following previous publications ${ }^{33-35}$. As such, FITC-dextran, a nonabsorbable molecule with $4.4 \mathrm{kDa}$ molecular mass (Sigma-Aldrich, St. Louis, MO, USA) at $12.5 \mathrm{mg}$ per mice was orally administered at $3 \mathrm{~h}$ before the detection of FITC-dextran in serum by Fluorospectrometer (NanoDrop 3300; Thermo Fisher Scientific, Wilmington, DE, USA). Serum endotoxin (LPS) was measured by HEK-Blue LPS Detection (InvivoGen, San Diego, CA, USA) and the data were recorded as 0 when LPS values were less than $0.01 \mathrm{EU} / \mathrm{ml}$ because of the limited lower range of the standard curve. Also, the cecum was used as a representative of the intestine to determine gut tight junction. Accordingly, cecum in Cryogel (Leica Biosystems, Richmond, IL, USA) were cut into $5 \mu \mathrm{m}$-thick frozen sections, fixed in acetone, blocked by blocking buffer, stained with a fluorescent antibody against ZO-1 and a green secondary antibody (Alexa Fluor 488) (Life Technologies, Carlsbad, CA, USA) before visualization and scoring by ZEISS LSM 800 (Carl Zeiss, Germany).

Analysis of mouse samples from blood, organs, and feces. After fasting for $12 \mathrm{~h}$ after free access to drinking water, lipid profiles were measured by the quantification assay for triglyceride (TG), total cholesterol (Sigma-Aldrich), low- and high- density lipoprotein cholesterol (LDL and HDL) (Crystal Chem Inc., Downers Grove, IL, USA). Liver damage and serum cytokines were determined by EnzyChrom Alanine Transaminase Assay (EALT-100; BioAssay Systems, Hayward, CA, USA) and enzyme-linked immunosorbent assays (ELISA) for mouse cytokines (Invitrogen, Carlsbad, CA, USA), respectively. For histology, paraffin-embedded sections 
(4 $\mu \mathrm{m}$ thick) stained by Hematoxylin and Eosin (H\&E) from $10 \%$ formalin-fixed samples were evaluated. The scoring system of obesity-induced liver damage was used as the following; steatosis (0-3), lobular inflammation $(0-3)$, and hepatocellular ballooning degeneration $(0-2)^{36}$. The thickness of subcutaneous fat was determined following a previous publication ${ }^{37}$. For the detection of lipids in the liver, livers were sonicated (High-Intensity Ultrasonic Processor, Newtown, CT, USA) in $500 \mu$ of ice-cold PBS containing protease inhibitor Cocktail (I3786) (Sigma-Aldrich) and measured lipids from the supernatant by the quantification assay for triglyceride and total cholesterol (Sigma-Aldrich).

In addition, oxidative stress in the liver was evaluated following a previous study ${ }^{38}$. Briefly, livers were homogenized in radioimmunoprecipitation assay (RIPA) with protease inhibitor Cocktail (I3786) (Sigma-Aldrich) on ice before measuring an oxidative stress molecule, malondialdehyde (MDA), (Cayman Chemical Company, Ann Arbor, MI, USA). For an anti-oxidant molecule, livers were sonicated in 2-(N-morpholino) ethanesulfonic acid (MES) buffer (Sigma-Aldrich) before the measurement of glutathione (GSH) (Cayman Chemical Company) from the supernatant. Furthermore, the mucin production from the colon was evaluated by real-time polymerase chain reaction (PCR). Briefly, the total RNA was prepared from the colon samples with an RNA-easy mini kit (Qiagen, Hilden, Germany), quantified by NanoDrop 100 Spectrophotometer (Thermo Fisher Scientific) before the determination of gene expression. Total RNA reverse transcription was processed with a High-Capacity cDNA Reverse Transcription (Thermo Fisher Scientific). Samples were performed using SYBR Green PCR Master Mix for quantitative real-time PCR with QuantStudio6 Flex Real-time PCR System (Thermo Fisher Scientific), respectively. The gene expression of Mucin-2 (MUC2) (forward 5'-CGACACCAGGGATTTCGCTTAAT-3'; reverse $5^{\prime}$-CACTTCCACCCTCCCGGCAAAC-3') were determined in terms of relative quantitation of the comparative threshold (delta-delta $\mathrm{Ct}$ ) method $(2-\Delta \Delta \mathrm{Ct})$ as normalized by $\beta$-actin (an endogenous housekeeping gene) (forward 5'-CGGTTCCGATGCCCTGAGGCTCTT-3'; reverse 5'-CGTCACACTTCATGATGGAATTGA-3').

Mouse fecal analysis and the ex vivo experiment on feces. Fecal $\mathrm{pH}$ was evaluated using $1 \mathrm{~g}$ of feces thoroughly mixed with $2 \mathrm{ml}$ of water before centrifugation at 4,000 rpm for $3 \mathrm{~min}$. Then, the $\mathrm{pH}$ of the supernatant was measured by $\mathrm{pH}$ meter (Orion 4-star, Benchtop pH/Conductivity Thermo Fisher Scientific). Besides, the bacterial abundance of Akkermansia muciniphila, the beneficial bacteria against obesity ${ }^{39}$, and Lactobacillus spp. in several segments of mouse intestines with fecal contents were evaluated by PCR. Total DNA was extracted by a QIAamp fast DNA Stool Mini Kit (Qiagen, Hiden, Germany), as per the manufacturer's instructions. Primers for the variable regions of the $16 \mathrm{~s}$ ribosomal RNA (rRNA) gene sequence of $A$. muciniphila bacteria $^{40}$ were: AM1, 5' - CAGCACGTGAAGGTGGGGAC-3'; AM2, 5'-CCTTGCGGTTGGCTCAGAT-3' and for L.rhamnosus ${ }^{41}$ were rham 5'-TGCATCTTGATTTAATTTTG-3'; Y2 5'-CCCACTGCTGCCTCCCGT AGGAGT-3'. The relative quantitation was normalized to the housekeeping $16 \mathrm{~S}$ bacterial rRNA gene (forward 5'-ACGCAACTGACGAGTGTGAC-3'; reverse, 5'-GATCGCGACACCGAACTAAT-3'). The comparative cycle threshold against the gene expression of these bacteria was demonstrated.

In addition, to further determine the association between Lactobacillus spp. and Akkermansia spp., cecal feces from 8 wk-HFD-administered mice $(30 \mathrm{mg}$ ) were anaerobically cultured under anaerobic conditions by gas generation sachets (AnaeroPack; Mitsubishi Gas Chemical, Japan) using Brain Heart infusion media (BHI; an enriched media for Akkermansia spp.) or De Man, Rogosa and Sharpe media (MRS; an enriched media for Lactobacillus spp.) (Oxoid Tryptone; Thermo Fisher Scientific) with or without LA5 for $48 \mathrm{~h}$ at $37^{\circ} \mathrm{C}$ before detecting bacterial gene expression by PCR as previously mentioned.

Fecal microbiome analysis. Feces from 9 mice ( 0.25 g per mouse) from different cages in each experimental group were divided into three samples per group ( 3 mice per sample) before performing microbiota analysis following a previous protocol ${ }^{42}$. In short, metagenomic DNA was extracted from $0.25 \mathrm{~g}$ feces by DNeasyPowerSoil Kit (Qiagen, MD, USA). The Universal prokaryotic 515F (forward; (5'-GTGCCAGCMGCCGCGGTAA-3') and 806R (reverse; 5'-GGACTACHVGGGTWTCTAAT-3'), with appended Illumina adapter and Golaybarcode sequences, were used for 16S rRNA gene V4 library construction and sequenced using Miseq 300 platform (Illumina, San Diego, CA, USA) at Omics Sciences and Bioinformatics Center, and Microbiome Research Unit for Probiotics in Food and Cosmetics, Chulalongkorn University. Raw sequences were quality processed and operational taxonomic unit (OTU) classified following Mothur'sstandard operating platform procedures ${ }^{43,44}$. Bioinformatic analyses included good's coverage, alpha diversity (e.g. Chao), and beta diversity (e.g. non-metric multidimensional scaling (NMDS)). Linear discriminant analysis Effect Size (LEfSe) and meta-stats were also performed to determine species marker and unique representing species of the interested group, respectively ${ }^{43,45}$. In addition, nucleic acid sequences in this study were deposited in an NCBI open-access sequence read archive database (SRA), accession number SRP286245.

In vitro experiment. Because serum endotoxin could be detected in obesity ${ }^{25}$ and saturated fatty acid-activated hepatocytes, the conditioned media from LA5 were incubated in HepG2 hepatocyte cell-line with palmitic acid (a representative saturated fatty acid) and/or endotoxin to see an impact of LA5 on hepatocytes. For the preparation of LA5 conditioned-media, LA5 at $0.1 \mathrm{McFarland}$ standards (OD600) were incubated anaerobically for $48 \mathrm{~h}$ before supernatant collection and filtered with a $0.22-\mu \mathrm{m}$ membrane filter (Minisart; Sartorius, Göttingen, Germany). Next, $500 \mu$ of the preparation was concentrated by speed vacuum drying at $40{ }^{\circ} \mathrm{C}$ for $3 \mathrm{~h}$ (Savant Instruments, NY) and the concentrated pellets were re-suspended with HyClone Dulbecco's Modified Eagle Medium (DMEM) high-glucose medium (HyClone; Laboratories, UT, USA). The small molecules of condition media were selected by the filtration with membrane filters (50 and $100 \mathrm{kDa}$ ) (Milipore; Merck, Darmstadt, Germany) and stored at $20^{\circ} \mathrm{C}$ before use. 
HepG2, a human hepatoma cell-line, (ATCC HB-8065; Thermo Fisher Scientific), was maintained in DMEM with $10 \%$ fetal bovine serum (FBS), $1 \%$ penicillin/streptomycin antibiotics, and $1 \%$ Sodium pyruvate in a humidified atmosphere of $5 \% \mathrm{CO}_{2}$ at $37^{\circ} \mathrm{C}$. HepG2 at $1 \times 10^{4}$ cells $/ \mathrm{ml}, 0.5 \mathrm{mM}$ of palmitic acid (PA) (Sigma-Aldrich), and $1 \mu \mathrm{g} / \mathrm{ml}$ of lipopolysaccharide (LPS from E. coli O55:B5) (Sigma-Aldrich) with or without LA5 conditioned media in 96-well plate with DMEM at $37^{\circ} \mathrm{C}$ before supernatant and cell collection. Supernatant cytokines were then determined using ELISA for human cytokines (TNF- $\alpha$, IL-8, and IL-10) (R\&D System, Minneapolis, MN, USA) and intracellular lipid content was determined by $0.3 \%$ Oil Red O staining (Sigma-Aldrich) with the evaluation by ImageJ (National Institute of Health, Bethesda, MD, USA) in 10 randomized fields ${ }^{46}$.

Statistical analysis. Mean \pm standard error (SE) was used for data presentation. The differences between groups were examined for statistical significance by one-way analysis of variance (ANOVA) followed by Tukey's analysis or Student's $t$-test for comparisons of multiple groups or 2 groups, respectively. All statistical analyses were performed with SPSS 11.5 software (SPSS, IL, USA) and Graph Pad Prism version 7.0 software (La Jolla, CA, USA). A $p$-value of $<0.05$ was considered statistically significant.

\section{Results}

Lactobacillus acidophilus LA5 attenuated obesity in high-fat diet mice. Lactobacillus acidophilus LA5 (LA5) attenuated obesity in mice as determined by bodyweight and visceral fat deposition in several sites including mesentery, retro-peritonium, peri-gonadal, peri-renal and subcutaneous fat along with lipid profiles (TG, total cholesterol, HDL and LDL) (Fig. 1A-K) supported several studies that utilizing LA5-combined probiotics ${ }^{26,27}$. In addition, LA5 also attenuated obesity-induced liver injury as evaluated by liver enzyme, liver weight, the histological score of steatohepatitis, the lipid composition of livers, and the oxidative stress (Fig. 2A$\mathrm{H})$. Moreover, LA5 improved gut permeability defect (gut leakage) and leaky gut-induced systemic inflammation as indicated by FITC-dextran assay, ZO-1tight junction protein, endotoxemia, colon mucin production (gene expression of $m u c 2$ ) and serum cytokines (TNF- $\alpha$, IL-6, and IL-10) (Fig. 3A-H). Because (i) Gram-negative bacteria in the gut is a source of endotoxin (LPS) ${ }^{7}$, (ii) obesity enhances endotoxemia from gut translocation ${ }^{25}$ and (iii) molecules from gut translocation is possibly directly transported to the liver through portal veins ${ }^{7}$, the reduced serum endotoxin in LA5 administered-obese mice implies the improved gut dysbiosis.

Lactobacillus acidophilus LA5 attenuated gut dysbiosis and promoted beneficial Akkermansia spp. in high-fat diet mice. From fecal microbiome analysis, HFD increased Proteobacteria, the pathogenic Gram-negative bacteria, without causing a difference in Bacteroides, the most abundance Gram-negative bacteria in feces, nor the total Gram-negative bacteria in feces when compared with the regular diet group (Figs. 4A, 5A-G). However, at a genus level, bacteria in the Bacteroidales group such as S24-7, AF-12, and Prevotella spp. were predominant in HFD mice compared with the regular diet group (Fig. 4A lower and Table 1), supporting obesity-enhanced Bacteroides bacteria as previously reported ${ }^{47}$. Interestingly, HFD induced endotoxemia (Fig. 1J), despite the similar Gram-negative bacterial burdens in feces (fecal LPS burdens) compared with regular diet mice (Fig. 5G), was the consequence of gut mucosal injury by pathogenic bacteria such as Desulfovibriospp. and RF32 $2^{48,49}$ in Proteobacteria group (Table 1). This implies a difference between fecal microbiota in the genus level of these groups. The microbiota analysis among regular diet, HFD, and HFD + LA5 groups are shown in Tables 1, 2, and 3.

In LA5 administration with HFD, there was an increase in Firmicutes (beneficial Gram-positive anaerobes), Verrucomycobia (Gram-positive anaerobes with some benefits) and Cyanobacteria (bacteria that has not been possible to isolate $)^{50-52}$ with a decrease in Bacteroides and total Gram-negative bacterial burdens in feces (Figs. 4A and 5A-G). The reduced fecal burdens of total Gram-negative bacteria (Fig. 5G) may be partly responsible for less severe endotoxemia in LA5-treated HFD mice (Fig. 1J). Interestingly, LA5 administration reduced Gammaproteobacteria, a group of pathogenic bacteria, including Pseudomonas spp., Klebsiella spp., and Escherichia coli, which also might be beneficial (Tables 2, 3). Additionally, the Linear discriminant analysis Effect Size (LEfSe) demonstrates the variation in bacteria among groups including (i) Helicobacter, Odoribacter, and Prevotella in the regular diet group, (ii) Bacteroides and Proteobacteria in HFD, and iii) Helicobacter, Desulfovibrio, Akkermansia, and Clostridium in HFD with LA5 (Fig. 4B). Moreover, HFD increased bacterial diversity as determined by Chao-1 richness estimation (variety of species) with similar evenness estimation (proportions of the individual species) in comparison to regular diet mice (Fig. 4C). Furthermore, the difference in fecal microbiome was demonstrated by a distinct separation in non-metric multidimensional scaling (NMDS) of bacteria based on the species taxonomic level (Fig. 4D). Notably, HFD increased fecal $\mathrm{pH}$ possibly due to reduced short-chain fatty acids (SCFAs)-induced bacteria ${ }^{53}$ but the acidification property of LA5 restored fecal $\mathrm{pH}$ in HFD-administered mice (Fig. 5H).

Among all of these bacteria enhanced by LA5 administration, Akkermansia muciniphila is known to attenuate obesity $^{39}$. Hence, we analyzed the presence of A. muciniphila and L. Acidophilus in the feces from various sections of the intestine by PCR. Without LA5, HFD mildly increased both bacteria only in the colon at approximately five-tenfold of the regular diet group (Fig. 6A-D). On the other hand, both bacteria were profoundly elevated in the jejunum, ileum, and colon, but not the duodenum in a regular diet or HDF with LA5 administration (Fig. 6A-D) implying the promotion of A. muciniphila by LA5. Notably, A. muciniphila in non-obese regular diet mice with LA5 was lower than HFD with LA5 (Fig. 6A-D) suggesting an influence of obesity with LA5 on A. muciniphila. Furthermore, the ex vivo incubation of feces from cecum with or without LA5 demonstrated the increased Akkermansia spp. as detected by PCR with LA5 incubation in either the enriched media for Lactobacilli (MRS) or the media for Akkermansia (BHI) (Fig. 6E,F). After incubation, the pH of the samples with LA5 demonstrated a lower $\mathrm{pH}$ than non-LA5 samples (Fig. 6G) implying a possible $\mathrm{pH}$-associated promotion of 

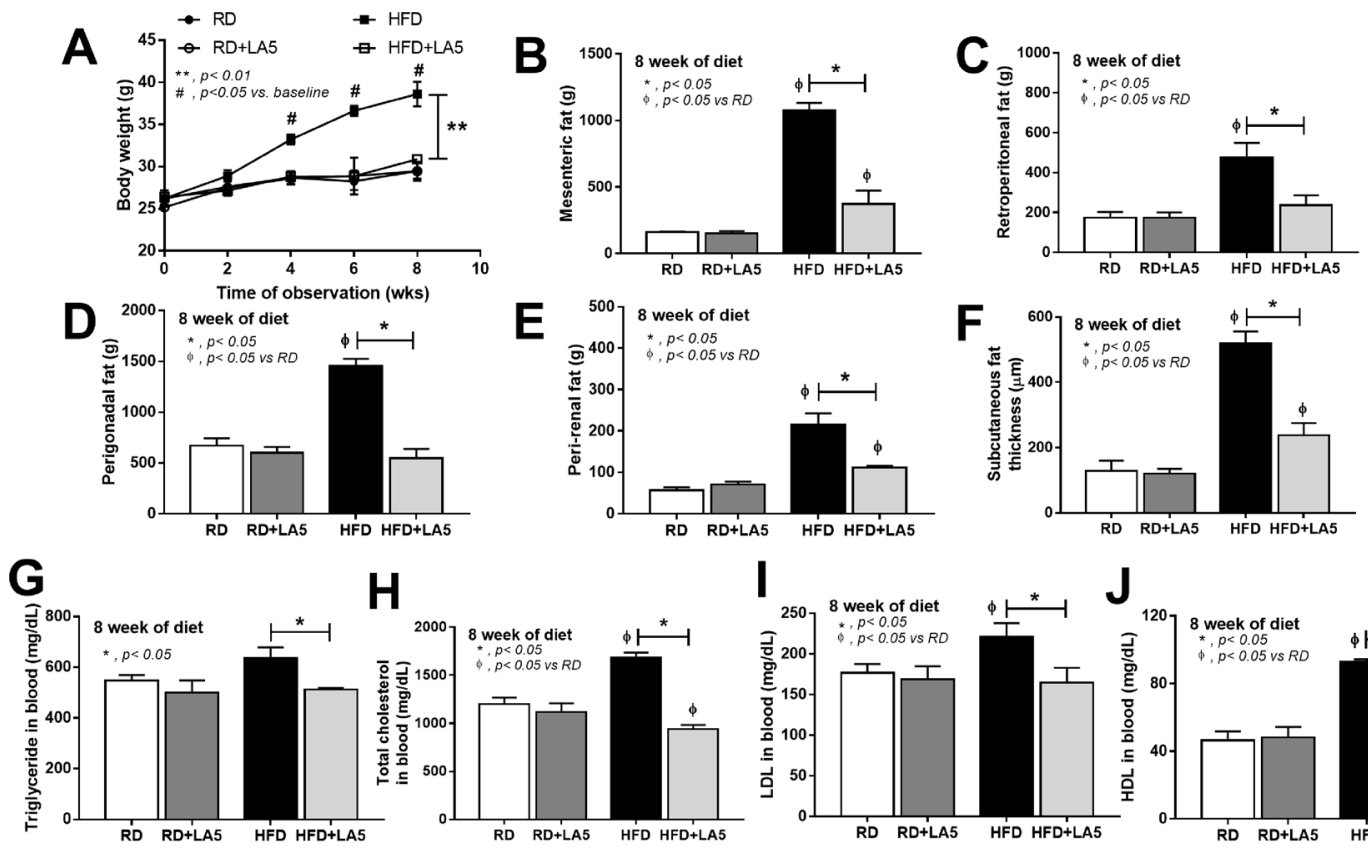

H
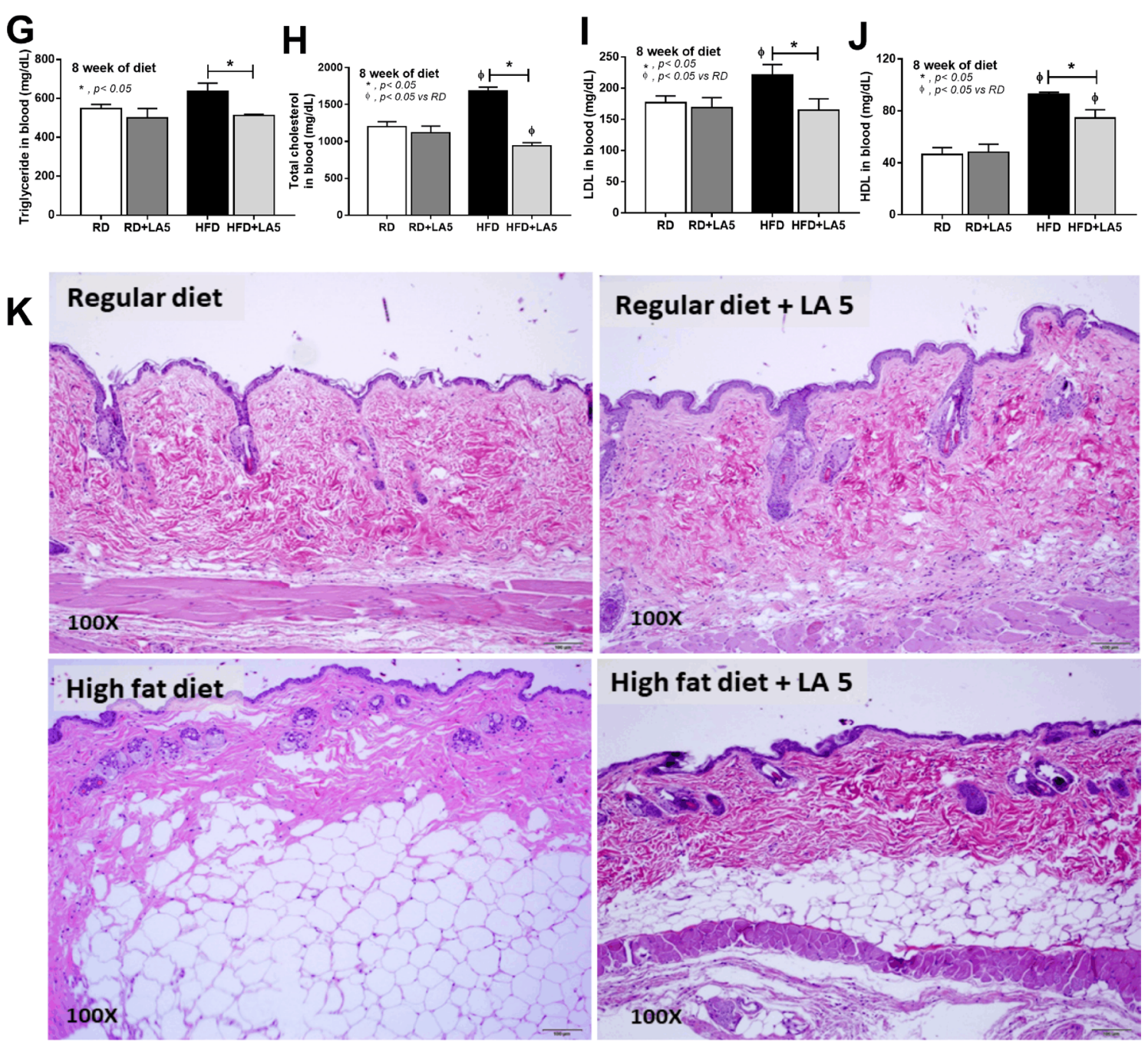

Figure 1. Characteristics of mice fed with regular diet (RD) or high-fat diet (HFD) with or without Lactobacillus acidophilus LA5 (LA5) as determined by body weight (A), adipose tissue depots in several sites (B-E), subcutaneous fat thickness (F), fasting blood lipid profiles $(\mathbf{G}-\mathbf{J})$ and the representative figures of the subcutaneous fat thickness (original magnification $\times 200)(\mathbf{K})$ were demonstrated $(n=6-8 /$ group for $\mathbf{A}-\mathbf{J})$. LDL, low-density lipoprotein; HDL, high-density lipoprotein.

Akkermansia growth (maximized growth at $\mathrm{pH} 6.5)^{54}$. Accordingly, the abundance of Akkermansia in the samples with LA5 co-incubation in MRS, an enriched media for Lactobacilli, was even higher than the abundance in BHI, an Akkermansia-enriched media, without LA5 (Fig. 6E,F).

Lactobacillus acidophilus LA5 attenuates fatty acid-induced hepatocyte inflammation. Because direct activation of LPS and Lactobacilli-producing molecules on hepatocytes during obesity- 


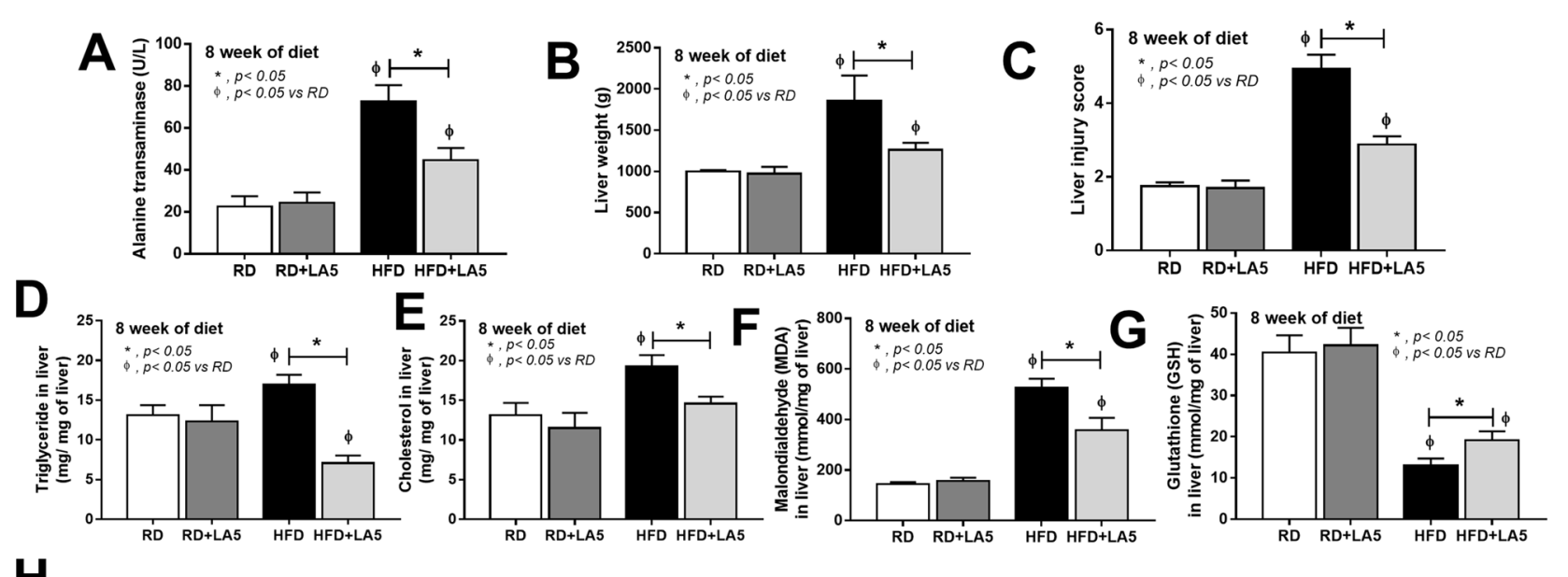

H
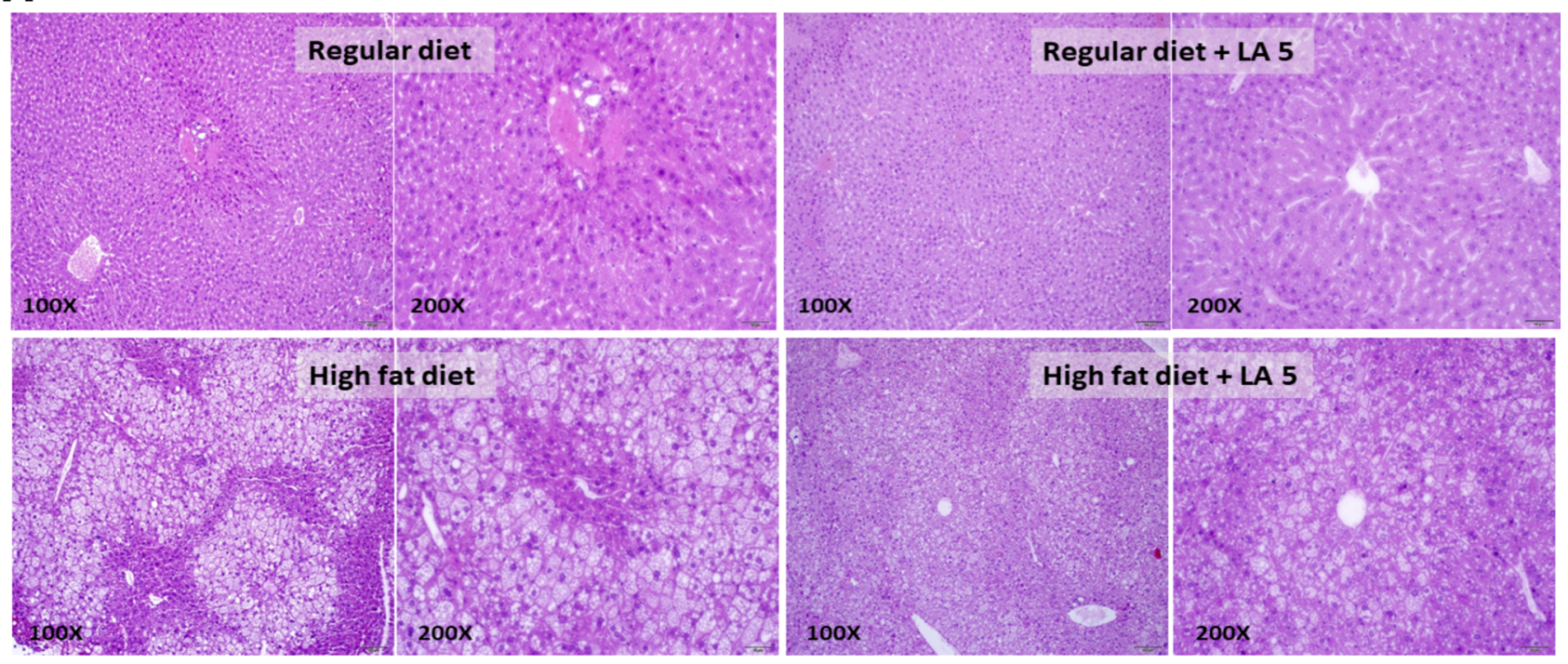

Figure 2. Characteristics of liver injury in mice fed with regular diet (RD) or high-fat diet (HFD) with or without Lactobacillus acidophilus LA5 (LA5) as determined by alanine transaminase (ALT) (A), liver weight (B), histological liver injury score (C), lipid components in the liver including triglyceride, total cholesterol (D,E), oxidative stress (malondialdehyde; MDA) in the liver (F), an anti-oxidant molecule (glutathione) in the liver (G) and the representative pictures of liver histology $(\mathbf{H})$ were demonstrated ( $n=6-8 /$ group for $\mathbf{A}-\mathbf{G})$. Severe lipid accumulation (steatohepatitis) was indicated in HFD mice.

induced gut translocation through portal vein is possible 7,55 , in vitro LPS activation on hepatocytes with or without Lactobacillus condition media was performed. Indeed, Lactobacillus condition media with molecular weight $>50$ and $<100 \mathrm{kDa}$-but not $<50$ and $>100 \mathrm{kDa}$-reduced cytokine production from HepG2 cells at $72 \mathrm{~h}$ post-incubation (Fig. 7A-C). Thus, Lactobacillus condition media with molecular weight $<100 \mathrm{kDa}$ was used in further experiments. On the other hand, palmitic acid (PA) induced lipid accumulation with mild supernatant cytokine production in comparison to LPS production, in HepG2 cells at $72 \mathrm{~h}$ post-incubation (Fig. 7D-F). The incubation of PA together withLPS (LPS + PA) induced similar cytokine responses to LPS activation alone and the LA5 supernatant was able to reduce the level of these cytokines at 48 and $72 \mathrm{~h}$ post-incubation (Fig. 7D-F). In parallel, the pre-incubation of PA for $24 \mathrm{~h}$ before LPS activation in HepG2 cells also enhanced supernatant cytokines and was attenuated by supernatant of LA5 (Fig. 7G-K) suggesting that LA5 produces anti-inflammatory substances.

\section{Discussion}

The administration of Lactobacillus acidophilus LA5 (LA5) together with a high-fat diet (HFD) attenuated hyperlipidemia, steatohepatitis, and obesity in a mouse model through the improved gut dysbiosis and the production of anti-inflammatory substances. Although only male mice were used for the experiments, these data were a proof of concept demonstrating the LA5 anti-obesity property.

Dysbiosis and metabolic endotoxemia in obese mice. The characteristics of obesity in HFD-administered mice were overweight, increased fat accumulation, hyperlipidemia, liver injury (liver weight, steatohepatitis, and elevated liver enzymes), and gut permeability defect (FITC-dextran assay, reduced tight junction 

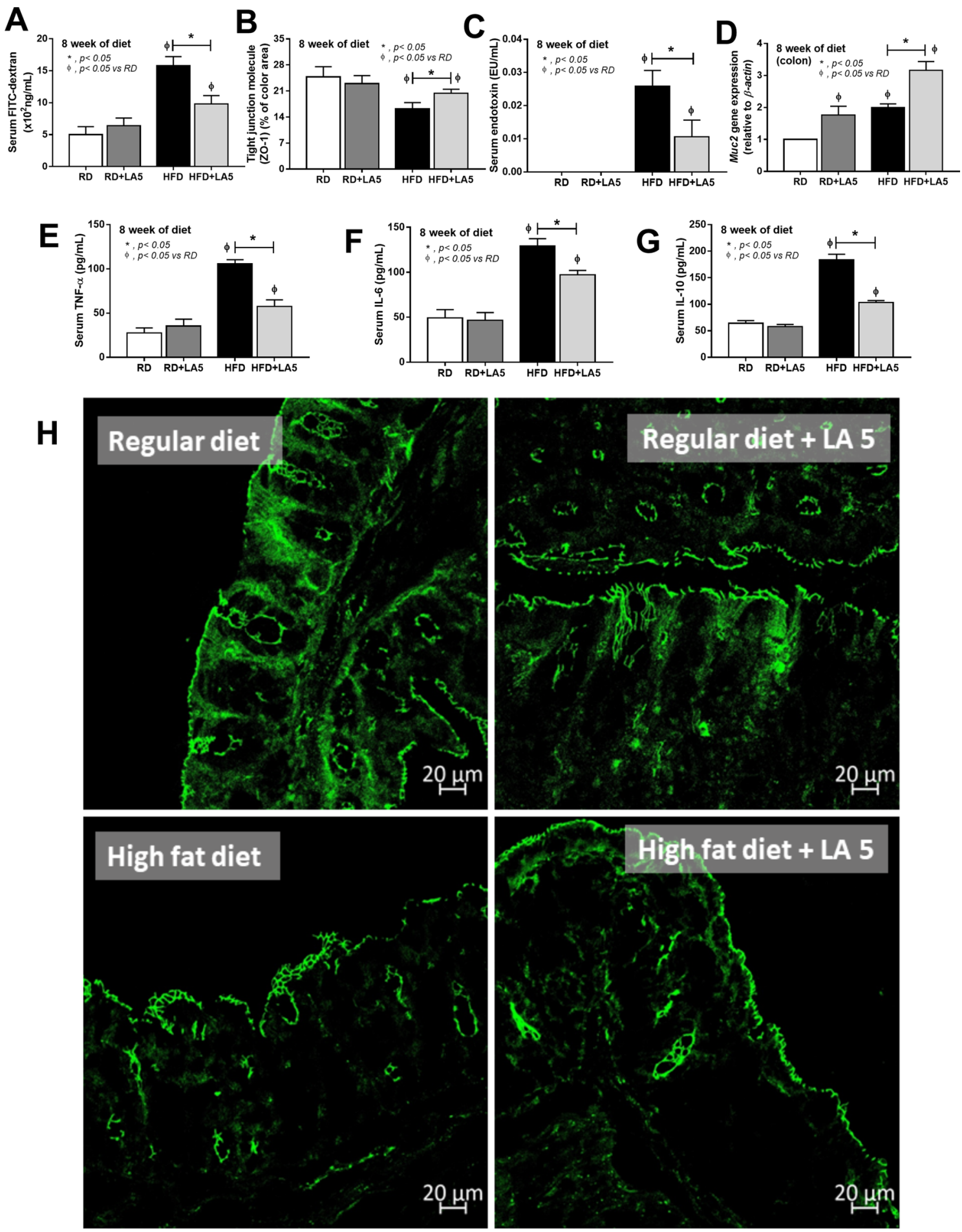

Figure 3. Characteristics of gut leakage and systemic inflammation in mice fed with regular diet (RD) or highfat diet (HFD) with or without Lactobacillus acidophilus LA5 (LA5) as determined by FITC-dextran assay (A), a tight junction protein (zonaoclludens-1; ZO-1) on intestinal mucosa (B), serum endotoxin $(\mathbf{C})$, gene expression of mucin-2 (muc2) in the colon (D), serum cytokines (E-G) and the representative fluorescent pictures of ZO-1 staining in cecal mucosa $(\mathbf{H})$ were demonstrated ( $n=6-8$ /group for $\mathbf{A}-\mathbf{G})$.

protein, and increased serum LPS). Obesity-induced liver injury is partly stimulated by a direct LPS activation on hepatocytes as a result of gut permeability defects ${ }^{7}$. Because inflammatory responses against pathogens (eg. LPS) are much stronger than the responses toward self-antigens ${ }^{6}$, obesity-induced endotoxemia might be a fundamental activator that leads to major complications ${ }^{56}$. Indeed, HFD enhances fecal basification (increased fecal $\mathrm{pH}$ ) possibly through amplified bile production and reduction of short-chain fatty acids (SCFAs ${ }^{57}$, which increase Proteobacteria (mucosal invasive Gram-negative organisms ${ }^{58,59}$ ) and enhance Deferribacteres (rodent 

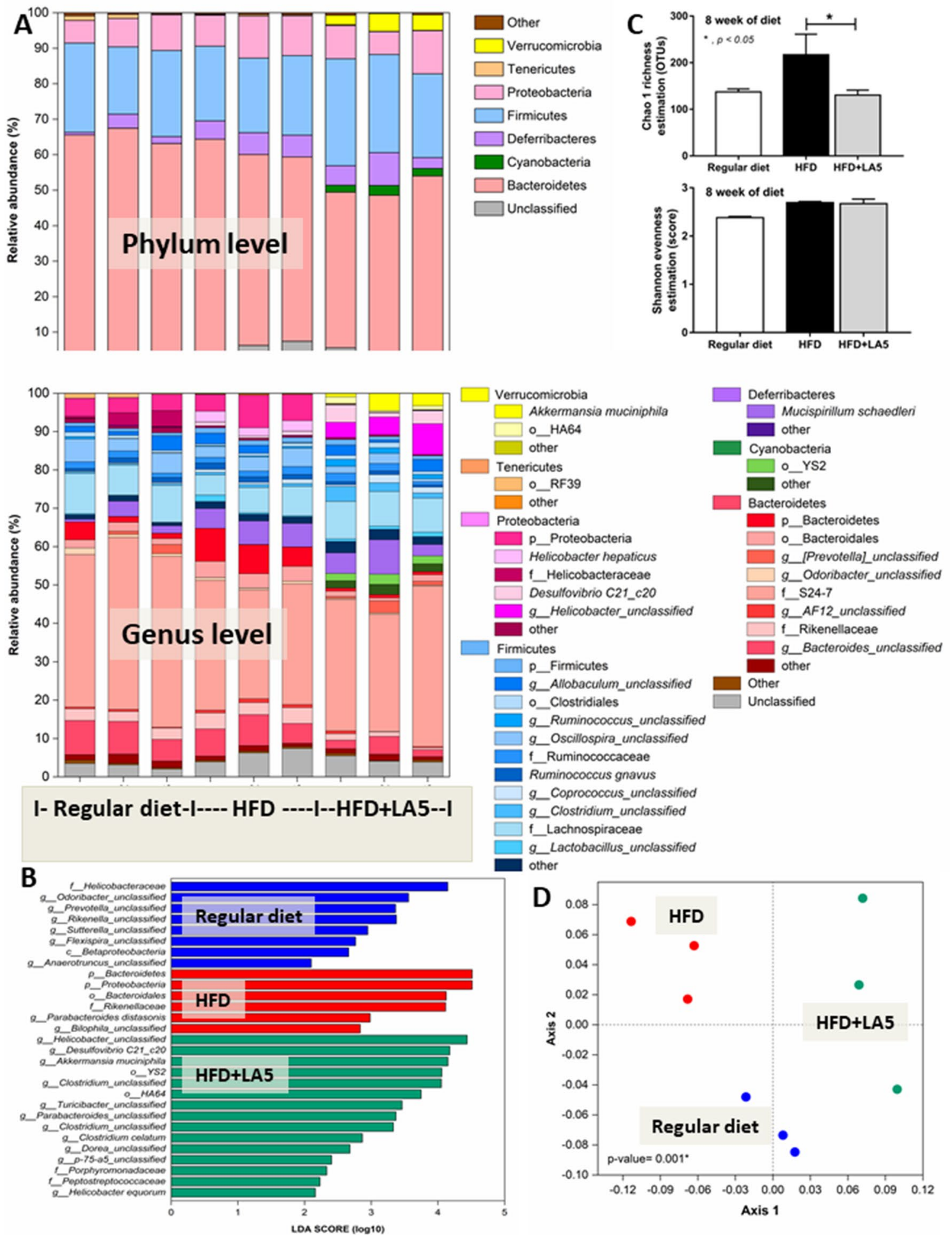

Figure 4. Gut microbiota analysis from feces of mice fed with a regular diet, high-fat diet (HFD), and HFD with Lactobacillus acidophilus LA5 (LA5) as determined by the relative abundance of bacterial diversity at phylum and genus (A), the possibly unique bacteria in each group by Linear discriminant Effect Size (LEfSe) analysis (B), the alpha diversity by Chao 1 richness estimation and Shannon evenness analysis (C) and nonmetric multidimensional scaling (NMDS) of bacteria based on the species taxonomic level (D).

gut-pathogens ${ }^{60}$ ) but not Bacteroidetes (Gram-negative anaerobes in pathogenic conditions ${ }^{61}$ ) nor fecal burdens of total Gram-negative bacteria. This data supports that HFD enhances endotoxemia ${ }^{62}$ through dysbiosis- 

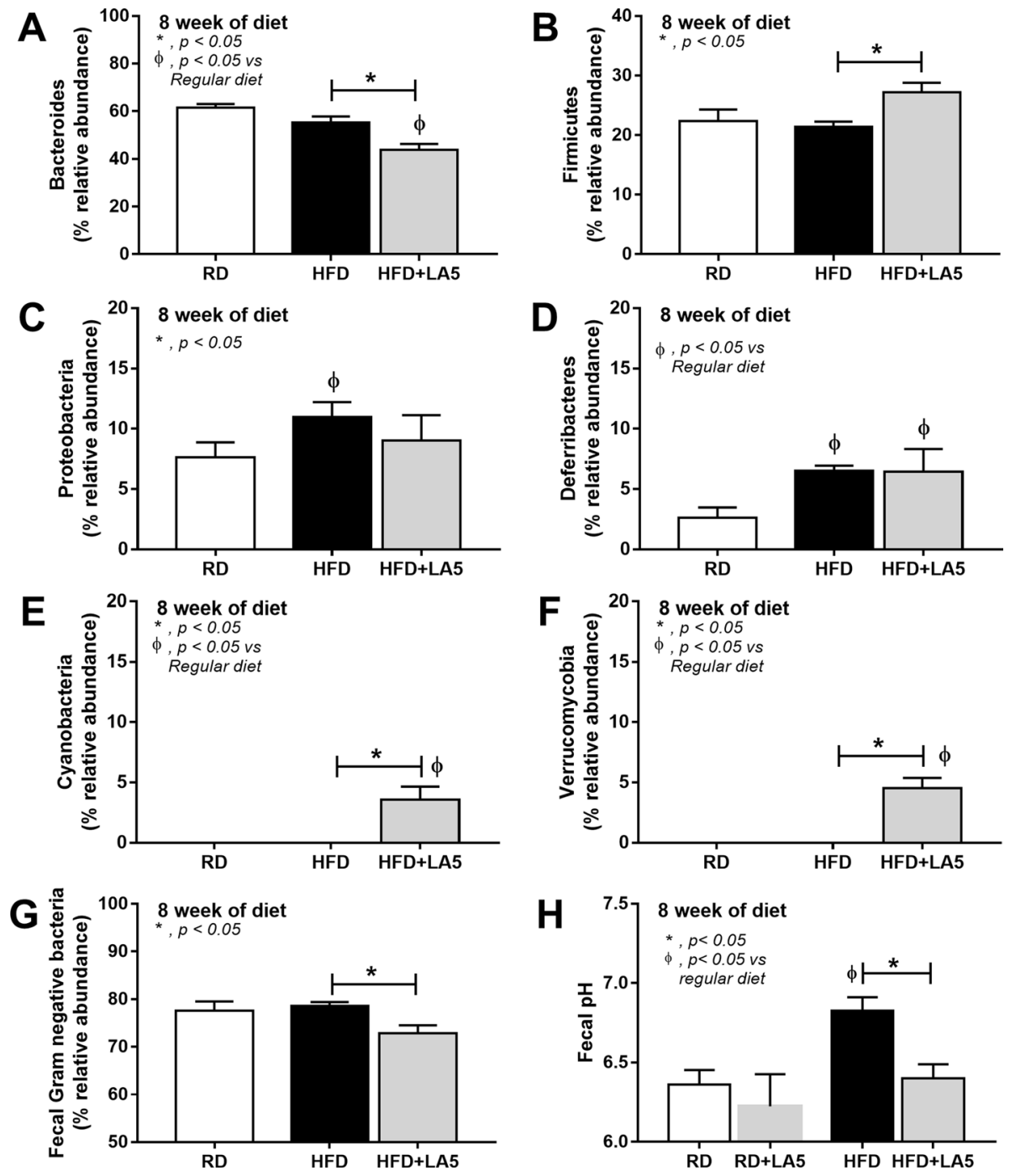

Figure 5. Gut microbiota and fecal analysis from feces of mice fed with a regular diet, high-fat diet (HFD), and HFD with Lactobacillus acidophilus LA5 (LA5) as determined by the relative abundance of bacterial diversity at phylum with bar graph presentation $(\mathbf{A}-\mathbf{F})$, total Gram-negative bacterial abundance in feces $(\mathbf{G})$ and fecal $\mathrm{pH}$ (H) were demonstrated.

induced intestinal mucosal injury ${ }^{63,64}$, but not increases LPS burdens in the gut, and the endotoxemia enhances liver injury and promotes systemic inflammation. Hence, the attenuation of dysbiosis and/ or gut-leakage may be a direct adjunctive treatment against obesity-induced inflammation and other complications.

Lactobacillus acidophilus LA5 attenuated obesity and gut dysbiosis. Probiotics are the most common treatment for gut dysbiosis ${ }^{22,24,25,50}$, attenuating obesity through several mechanisms such as the induction of energy-efficient microbiota, production of SCFAs, and promotion of intestinal hormones with anti-obesity properties $^{65}$. Among several probiotics, Lactobacillus acidophilus demonstrates robust lactic acid production in comparison to other strains ${ }^{66}$ that may strongly alter HFD-induced dysbiosis. The non-difference in Lachnospiraceae bacteria (Lactobacillus group) in fecal microbiome analysis (Fig. 4A) between mice with versus without LA5 administration demonstrated the intestinal attachment property of a good probiotics ${ }^{67}$. Indeed, LA5 was able to normalize fecal $\mathrm{pH}$, decrease Bacteroidetes, a predominant group of bacteria in pathogenic conditions $\mathrm{s}^{61}$, reduce total Gram-negative bacterial strain in feces as well as attenuate endotoxemia. Additionally, LA5 enhanced Firmicutes (Gram-positive bacteria that prominently identified in healthy gut ${ }^{68}$ ) and Verrucomycobia (a phylum of bacteria that inversely correlated to several intestinal abnormalities ${ }^{54}$ ) in feces that possibly attenuated dysbiosis and improved intestinal mucosal integrity. Interestingly, Akkermansia muciniphila is a bacterium of the phylum Verrucomicrobia that demonstrates several advantages including anti-obesity properties ${ }^{30,69}$. While the intestinal mucus layer in obese mice is reduced by $\mathrm{HFD}^{70}$, A. muciniphila increases the intestinal mucin thickness and attenuates gut-leakage ${ }^{71}$, despite having a mucin-degrading function ${ }^{40}$. When LA5 was administered, A. muciniphila in the colon increased by 2,000 folds (as determined by bacterial nucleic acid) in comparison to the control group, alluding to another mechanism of gut-leakage attenuation. 


\begin{tabular}{|l|c|c|l|l|}
\hline \multirow{2}{*}{ Bacteria } & \multicolumn{2}{|l|}{$\begin{array}{l}|l| \\
(\%)\end{array}$} & \multirow{2}{*}{} \\
\cline { 2 - 4 } & Regular diet & HFD & p-value & Direction \\
\hline g_Bilophila_unclassified & 0.000 & 0.135 & 0.002 & Up \\
\hline Helicobacter hepaticus & 0.003 & 2.547 & 0.003 & Up \\
\hline p__Firmicutes & 0.814 & 1.351 & 0.004 & Up \\
\hline o__Bacteroidales & 2.009 & 3.927 & 0.005 & Up \\
\hline g__Clostridium_unclassified & 0.001 & 0.158 & 0.007 & Up \\
\hline f_Porphyromonadaceae & 0.000 & 0.014 & 0.009 & Up \\
\hline Desulfovibrio C21_c20 & 0.059 & 0.855 & 0.012 & Up \\
\hline Parabacteroides distasonis & 0.000 & 0.184 & 0.023 & Up \\
\hline Mucispirillum schaedleri & 2.376 & 5.842 & 0.025 & Up \\
\hline g__AF12_unclassified & 0.411 & 0.882 & 0.026 & Up \\
\hline o__RF32 & 0.004 & 0.043 & 0.030 & Up \\
\hline g__Coprococcus_unclassified & 0.253 & 0.812 & 0.038 & Up \\
\hline p__Bacteroidetes & 2.276 & 6.975 & 0.039 & Up \\
\hline unclassified & 2.830 & 5.977 & 0.043 & Up \\
\hline f_Lactobacillaceae & 0.016 & 0.051 & 0.047 & Up \\
\hline f_Rikenellaceae & 2.835 & 3.789 & 0.048 & Up \\
\hline g_Prevotella_unclassified & 0.530 & 0.063 & 0.001 & Down \\
\hline f_S24-7 & 43.375 & 31.063 & 0.008 & Down \\
\hline c_BBetaproteobacteria & 0.095 & 0.017 & 0.029 & Down \\
\hline
\end{tabular}

Table 1. Comparison of fecal microbiota in genus level between regular diet vs high fat diet (HFD) mice. ${ }^{*} \mathrm{p}<0.05$ Regular diet vs HFD. ${ }^{*}$ Direction of change in HFD compared with regular diet.

\begin{tabular}{|c|c|c|c|c|}
\hline \multirow[b]{2}{*}{ Bacteria } & \multicolumn{2}{|c|}{$\begin{array}{l}\text { Relative abundance } \\
(\%)\end{array}$} & \multirow[b]{2}{*}{ p-value ${ }^{*}$} & \multirow[b]{2}{*}{ Direction } \\
\hline & HFD & HFD + LA5 & & \\
\hline o__YS2 & 0.015 & 2.226 & 0.002 & Up \\
\hline o__Desulfovibrionales & 0.011 & 0.273 & 0.005 & $\mathrm{Up}$ \\
\hline f_Peptostreptococcaceae & 0.001 & 0.031 & 0.008 & Up \\
\hline g__Dorea_unclassified & 0.015 & 0.106 & 0.012 & Up \\
\hline f__Porphyromonadaceae & 0.014 & 0.036 & 0.014 & Up \\
\hline g__Helicobacter_unclassified & 0.081 & 5.448 & 0.019 & $\mathrm{Up}$ \\
\hline f__Lachnospiraceae & 6.560 & 9.502 & 0.028 & $\mathrm{Up}$ \\
\hline g__p-75-a5_unclassified & 0.000 & 0.051 & 0.041 & $\mathrm{Up}$ \\
\hline g_Prevotella_unclassified & 0.063 & 0.179 & 0.043 & $\mathrm{Up}$ \\
\hline g__Parabacteroides_unclassified & 0.005 & 0.470 & 0.049 & Up \\
\hline Helicobacter hepaticus & 2.547 & 0.005 & 0.001 & Down \\
\hline o__Bacteroidales & 3.927 & 1.325 & 0.003 & Down \\
\hline $\mathrm{p}$ _Bacteroidetes & 6.975 & 0.826 & 0.006 & Down \\
\hline $\mathrm{p}$ __Proteobacteria & 6.687 & 0.084 & 0.009 & Down \\
\hline f__Rikenellaceae & 3.789 & 1.237 & 0.010 & Down \\
\hline g_Ruminococcus_unclassified & 0.001 & 0.000 & 0.016 & Down \\
\hline g__Odoribacter_unclassified & 0.609 & 0.298 & 0.023 & Down \\
\hline c__Gammaproteobacteria & 0.020 & 0.004 & 0.024 & Down \\
\hline [Ruminococcus] gnavus & 1.417 & 0.794 & 0.030 & Down \\
\hline Parabacteroides distasonis & 0.184 & 0.002 & 0.031 & Down \\
\hline g__Alistipes_unclassified & 0.161 & 0.037 & 0.038 & Down \\
\hline g_Bacteroides_unclassified & 6.720 & 2.861 & 0.045 & Down \\
\hline Proteus myxofaciens & 0.002 & 0.000 & 0.047 & Down \\
\hline
\end{tabular}

Table 2. Comparison of fecal microbiota in genus level between high fat diet (HFD) vs probiotics-treated HFD (HFD + LA5) mice. ${ }^{*} \mathrm{p}<0.05$ HFD vs HFD + LA5. ${ }^{*}$ Direction of change in HFD + LA5 compared with HFD. 


\begin{tabular}{|c|c|c|c|c|}
\hline \multirow[b]{2}{*}{ Bacteria } & \multicolumn{2}{|c|}{ Relative abundance (\%) } & \multirow[b]{2}{*}{ p-value ${ }^{*}$} & \multirow[b]{2}{*}{ Direction } \\
\hline & Regular diet & HFD + LA5 & & \\
\hline g__Clostridium_unclassified & 0.427 & 2.771 & 0.003 & Up \\
\hline o__YS2 & 0.010 & 2.226 & 0.004 & Up \\
\hline o__Desulfovibrionales & 0.006 & 0.273 & 0.009 & Up \\
\hline f__Peptostreptococcaceae & 0.000 & 0.031 & 0.010 & Up \\
\hline g__Lactobacillus_unclassified & 0.194 & 0.893 & 0.015 & Up \\
\hline g__Ruminococcus_unclassified & 0.220 & 1.543 & 0.018 & Up \\
\hline g__Helicobacter_unclassified & 0.141 & 5.448 & 0.019 & Up \\
\hline Lactobacillus reuteri & 0.000 & 0.136 & 0.023 & Up \\
\hline Alistipes finegoldii & 0.002 & 0.015 & 0.034 & Up \\
\hline Desulfovibrio C21_c20 & 0.059 & 3.329 & 0.038 & Up \\
\hline g__Parabacteroides_unclassified & 0.002 & 0.470 & 0.043 & Up \\
\hline g__Clostridium_unclassified & 0.001 & 0.442 & 0.045 & Up \\
\hline p__Proteobacteria & 4.243 & 0.084 & 0.001 & Down \\
\hline g_Sutterella_unclassified & 0.196 & 0.028 & 0.002 & Down \\
\hline g_Prevotella_unclassified & 0.530 & 0.179 & 0.007 & Down \\
\hline Proteus myxofaciens & 0.010 & 0.000 & 0.008 & Down \\
\hline o__Burkholderiales & 0.006 & 0.000 & 0.013 & Down \\
\hline g_Flexispira_unclassified & 0.105 & 0.000 & 0.020 & Down \\
\hline c_Betaproteobacteria & 0.095 & 0.004 & 0.021 & Down \\
\hline g__Alistipes_unclassified & 0.123 & 0.037 & 0.022 & Down \\
\hline c__Gammaproteobacteria & 0.005 & 0.004 & 0.023 & Down \\
\hline g__Bacteroides_unclassified & 7.581 & 2.861 & 0.029 & Down \\
\hline g_Oscillospira_unclassified & 4.676 & 1.767 & 0.034 & Down \\
\hline g__Desulfovibrio_unclassified & 0.296 & 0.004 & 0.036 & Down \\
\hline Butyricicoccus pullicaecorum & 0.148 & 0.070 & 0.044 & Down \\
\hline
\end{tabular}

Table 3. Comparison of fecal microbiota in genus level between regular diet vs probiotics-treated HFD (HFD + LA5) mice. ${ }^{*} \mathrm{p}<0.05$ Regular diet vs HFD + LA5. " Direction of change in HFD + LA5 compared with Regular diet.

Interestingly, the optimal $\mathrm{pH}$ for Akkermansia growth is $6.5^{54}$ but the $\mathrm{pH}$ of HFD feces was higher than 6.5 (Fig. $5 \mathrm{H}$ ) possibly due to the reduction of short-chain fatty acids ${ }^{53}$. Indeed, an abundance of Akkermansia spp. in fecal samples with LA5 using MRS media (the Lactobacilli enriched media) was higher than the culture in BHI (the Akkermansia enriched media) (Fig. 6E-G) supporting an impact of pH on Akkermansia growth. Although Lactobacilli might produce several promoting factors for Akkermansia growth, the acidification property of Lactobacilli might be one of these factors. On the other hand, in non-obese regular diet mice, LA5 also increased A. muciniphila (without fecal acidification) and induced colon mucin production ( $m u c 2$ gene expression) supported LA5 benefit on the enhanced-intestinal mucosal integrity through $A$. muciniphila induction in the healthy host. Meanwhile, the lower A. muciniphila in LA5-administered non-obese mice than the LA5-administered obese mice, despite the none A. muciniphila in HFD mice without LA5, implied the different mechanisms of LA5-enhanced $A$. muciniphila between obese mice versus non-obese mice possibly through fecal acidification versus some fat derivatives, respectively. Hence, the beneficial effects of probiotics in healthy and unhealthy mice might be different. Unfortunately, the fecal microbiome analysis in feces from LA5-administered regular diet mice (non-obese mice) was not performed here. Nevertheless, the LA5 administration in obesity might be an interesting growth promotion of the beneficial bacteria that are difficult to cultivate including $A$. muciniphila ${ }^{30}$. More studies are interesting.

Lactobacillus acidophilus LA5 attenuated hepatic injury through the production of anti-inflammatory molecules. Although molecules with a molecular weight (MW) larger than $600 \mathrm{Da}$ are kept inside the gut by intestinal integrity ${ }^{7}$, gut leakage allows these larger molecules to translocate through the gut. During gut leakage, beneficial molecules may be translocated alongside harmful molecules. For instance, LA5 produces anti-inflammatory molecules with MW of $50-100 \mathrm{kDa}$ - similar to the MW of LPS - that decreases supernatant cytokines of LPS-activated HepG2 cells via co-incubation or post-incubation with palmitic acid, a representative saturated fatty acid. Thus, hepatocytes may be activated by both LPS and anti-inflammatory molecules from LA5 from gut leakage. Further studies into the role of these molecules in reducing hepatocyte injury may yield fascinating results.

In conclusion, HFD induced gut dysbiosis, gut leakage resulted in endotoxemia and systemic inflammation (Fig. 8). Then LA5 administration directly adjusted gut microbiome, in part, by acidifying fecal $\mathrm{pH}$ that promoted Akkermansia spp. and directly attenuated hepatic injury through Lactobacilli producing anti-inflammatory molecules. Additionally, promoted Akkermansia spp. is an indirect anti-obesity effect of LA5 through the 

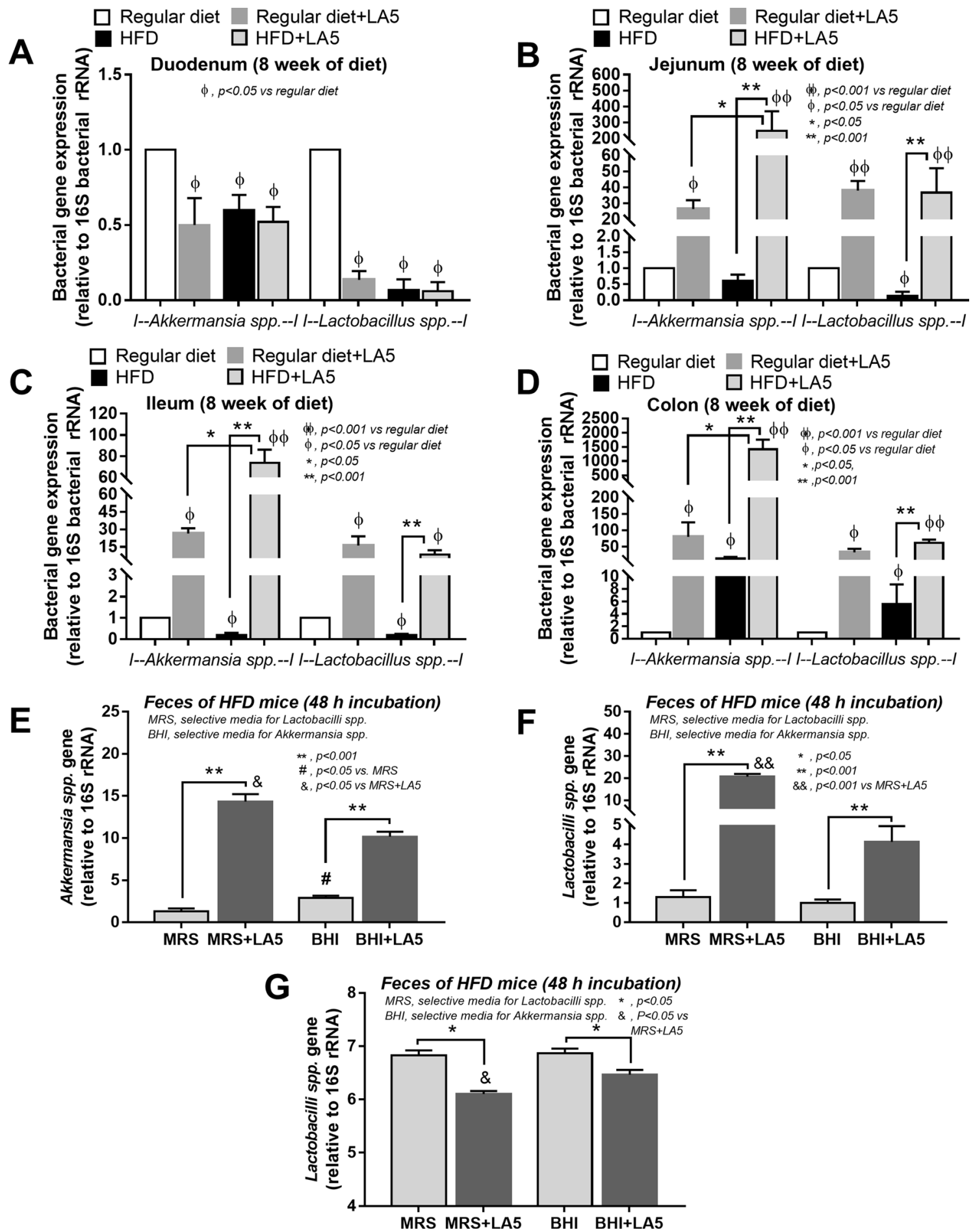

Figure 6. The abundance of bacterial gene expression in several parts of mouse intestines with fecal contents from mice fed with a regular diet, high-fat diet (HFD), and regular diet or HFD with Lactobacillus acidophilus LA5 (LA5) as determined by polymerase chain reaction (PCR) were demonstrated (A-D) ( $n=5-6 /$ group). Additionally, the abundance of bacterial gene expression in feces from HFD mice with or without LA5 after $48 \mathrm{~h}$ incubation in De Man, Rogosa, and Sharpe media (MRS; an enriched media for Lactobacilli) or Brain Heart Infusion media (BHI; an enriched media for Akkermansia) by PCR (E,F) and the pH of these samples (G) were demonstrated (independent triplicate experiments were performed).

known-mechanisms including improved energy consumption, produced SCFAs, gut leakage attenuation, reduced endotoxemia, and decreased liver injury ${ }^{39}$. Hence, the enhanced beneficial bacteria in the gut by probiotics is another interesting objective for probiotic utilization. 

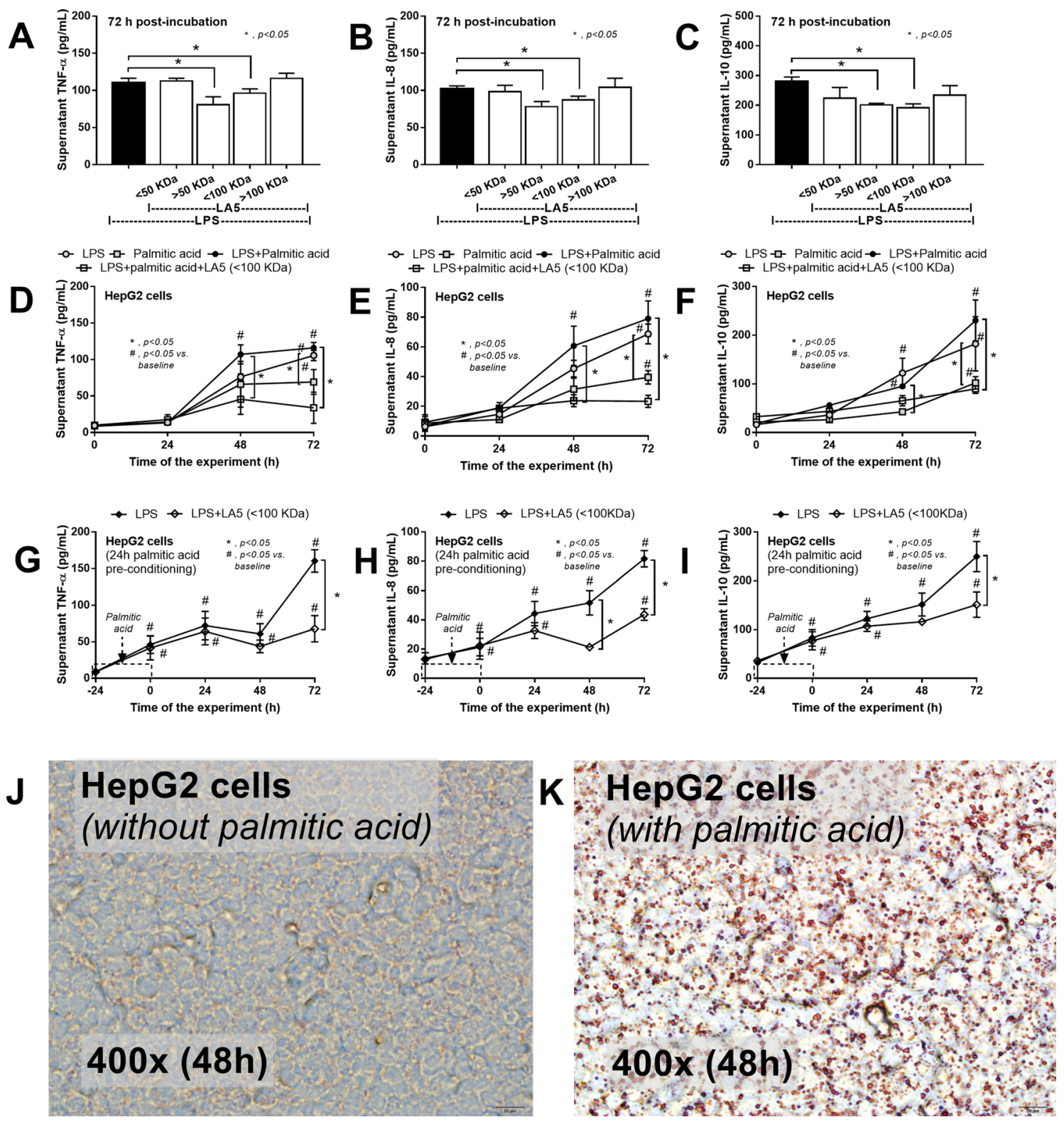

Figure 7. Supernatant cytokines (TNF- $\alpha$, IL-8, and IL-10) from HepG2 cells (hepatocytes) after activation by lipopolysaccharide (LPS) with condition media of Lactobacillus acidophilus LA5 (LA5) after filtered with membrane filters at 50 and $100 \mathrm{kDa}(\mathrm{A}-\mathrm{C})$, byLPS and palmitic acid (PA; a representative saturated fatty acid), alone or in combination, with or without LA5 condition media after filtered with $100 \mathrm{kDa}$ membrane filters (D-F) were demonstrated. Besides, the supernatant cytokines from LPS-activated HepG2 cells after $24 \mathrm{~h} \mathrm{PA}$ pre-conditioning with or without filtrated LA5 condition media $(100 \mathrm{kDa})(\mathbf{G}-\mathbf{I})$ and the representative pictures of HepG2 cell without or with PA incubation $(\mathbf{J}, \mathbf{K})$ were indicated (independent triplicate experiments were performed). 


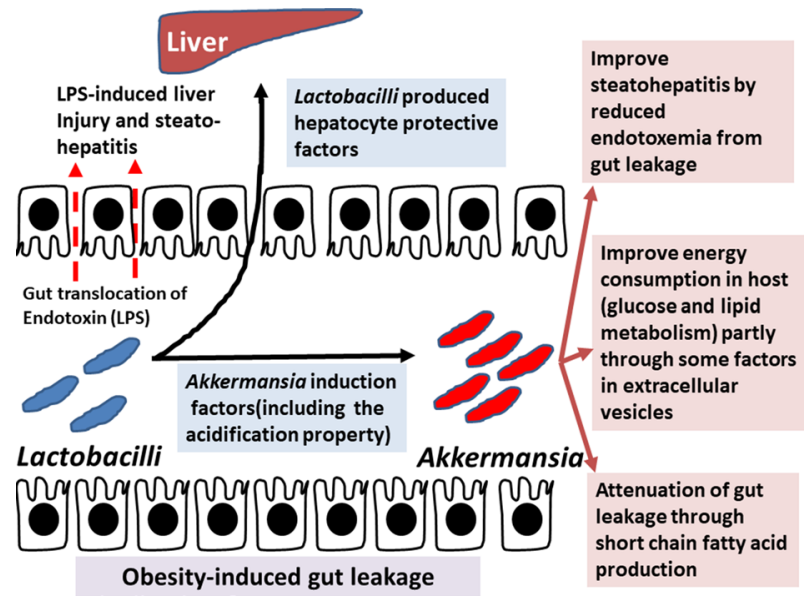

Figure 8. The proposed hypothesis demonstrates anti-obesity mechanisms of Lactobacillus acidophilus LA5 (LA5) directly through (i) promotion of Akkermansia spp. through an adjustment of fecal pH and (ii) attenuation of hepatic injury by gut translocation of the protective molecules. Besides, the known beneficial effects on obesity of LA5-promoted Akkermansia spp. including improved energy metabolism in the host, strengthened gut-permeability through short-chain fatty acids, and reduced hepatic injury by the attenuation of obesity-induced endotoxemia ${ }^{39}$ are the indirect anti-obesity effects of LA5.

Received: 15 October 2020; Accepted: 23 February 2021

Published online: 18 March 2021

\section{References}

1. Allison, D. B., Fontaine, K. R., Manson, J. E., Stevens, J. \& VanItallie, T. B. Annual deaths attributable to obesity in the United States. JAMA 282, 1530-1538. https://doi.org/10.1001/jama.282.16.1530 (1999).

2. Ross, P. A., Newth, C. J., Leung, D., Wetzel, R. C. \& Khemani, R. G. Obesity and mortality risk in critically ill children. Pediatrics 137, e20152035. https://doi.org/10.1542/peds.2015-2035 (2016)

3. Kolyva, A. S. et al. The role of obesity in the immune response during sepsis. Nutr. Diabetes 4, e137. https://doi.org/10.1038/nutd. 2014.34 (2014)

4. Singer, G., Stokes, K. Y., Terao, S. \& Granger, D. N. Sepsis-induced intestinal microvascular and inflammatory responses in obese mice. Shock 31, 275-279. https://doi.org/10.1097/SHK.0b013e3181834ab3 (2009).

5. McArdle, M. A., Finucane, O. M., Connaughton, R. M., McMorrow, A. M. \& Roche, H. M. Mechanisms of obesity-induced inflammation and insulin resistance: Insights into the emerging role of nutritional strategies. Front. Endocrinol. 4, 52 (2013).

6. Eppensteiner, J. et al. Damage- and pathogen-associated molecular patterns play differential roles in late mortality after critical illness. JCI Insight https://doi.org/10.1172/jci.insight.127925 (2019).

7. Amornphimoltham, P., Yuen, P. S. T., Star, R. A. \& Leelahavanichkul, A. Gut leakage of fungal-derived inflammatory mediators: Part of a gut-liver-kidney axis in bacterial sepsis. Dig. Dis. Sci. 64, 2416-2428. https://doi.org/10.1007/s10620-019-05581-y (2019).

8. Nagpal, R. et al. Obesity-linked gut microbiome dysbiosis associated with derangements in gut permeability and intestinal cellular homeostasis independent of diet. J. Diabetes Res. 2018, 3462092. https://doi.org/10.1155/2018/3462092 (2018).

9. Heisel, T. et al. High-fat diet changes fungal microbiomes and interkingdom relationships in the murine gut. $m S p h e r e$ https://doi. org/10.1128/mSphere.00351-17 (2017).

10. Murphy, E. A., Velazquez, K. T. \& Herbert, K. M. Influence of high-fat diet on gut microbiota: A driving force for chronic disease risk. Curr. Opin. Clin. Nutr. Metab. Care 18, 515-520. https://doi.org/10.1097/MCO.0000000000000209 (2015).

11. Leelahavanichkul, A. et al. Gastrointestinal leakage detected by serum (1->3)-beta-D-glucan in mouse models and a pilot study in patients with sepsis. Shock 46, 506-518. https://doi.org/10.1097/SHK.0000000000000645 (2016).

12. Zhang, Q. et al. Clinicopathological correlation of keratinocyte growth factor and matrix metalloproteinase-9 expression in human gastric cancer. Tumori 101, 566-571. https://doi.org/10.5301/tj.5000367 (2015).

13. Scaldaferri, F. et al. Gut microbial flora, prebiotics, and probiotics in IBD: Their current usage and utility. Biomed. Res. Int. 2013, 435268. https://doi.org/10.1155/2013/435268 (2013).

14. Hager, C. L. \& Ghannoum, M. A. The mycobiome: Role in health and disease, and as a potential probiotic target in gastrointestinal disease. Dig. Liver Dis. 49, 1171-1176. https://doi.org/10.1016/j.dld.2017.08.025 (2017).

15. Zuo, T. \& Ng, S. C. The gut microbiota in the pathogenesis and therapeutics of inflammatory bowel disease. Front. Microbiol. 9 , 2247. https://doi.org/10.3389/fmicb.2018.02247 (2018).

16. Hosono, A. et al. Characterization of a water-soluble polysaccharide fraction with immunopotentiating activity from Bifidobacterium adolescentis M101-4. Biosci. Biotechnol. Biochem. 61, 312-316. https://doi.org/10.1271/bbb.61.312 (1997).

17. Wu, M. H. et al. Exopolysaccharide activities from probiotic bifidobacterium: Immunomodulatory effects (on J774A.1 macrophages) and antimicrobial properties. Int. J. Food Microbiol. 144, 104-110. https://doi.org/10.1016/j.ijfoodmicro.2010.09.003 (2010).

18. Surayot, U. et al. Exopolysaccharides from lactic acid bacteria: Structural analysis, molecular weight effect on immunomodulation. Int. J. Biol. Macromol. 68, 233-240. https://doi.org/10.1016/j.ijbiomac.2014.05.005 (2014).

19. Gao, K. et al. Immunomodulation and signaling mechanism of Lactobacillus rhamnosus GG and its components on porcine intestinal epithelial cells stimulated by lipopolysaccharide. J. Microbiol. Immunol. Infect. 50, 700-713. https://doi.org/10.1016/j. jmii.2015.05.002 (2017).

20. Panpetch, W. et al. Lactobacillus rhamnosus L34 attenuates gut translocation-induced bacterial sepsis in murine models of Leaky Gut. Infect. Immunol. https://doi.org/10.1128/IAI.00700-17 (2018). 
21. Boonma, P., Spinler, J. K., Venable, S. F., Versalovic, J. \& Tumwasorn, S. Lactobacillus rhamnosus L34 and Lactobacillus casei L39 suppress Clostridium difficile-induced IL-8 production by colonic epithelial cells. BMC Microbiol. 14, 177. https://doi.org/10.1186/ 1471-2180-14-177 (2014).

22. Panpetch, W. et al. Additional Candida albicans administration enhances the severity of dextran sulfate solution induced colitis mouse model through leaky gut-enhanced systemic inflammation and gut-dysbiosis but attenuated by Lactobacillus rhamnosus L34. Gut Microbes https://doi.org/10.1080/19490976.2019.1662712 (2019).

23. Leelahavanichkul, A. et al. Evaluation of gastrointestinal leakage using serum $(1 \rightarrow 3)-\beta$-D-glucan in a Clostridium difficile murine model. FEMS Microbiol. Lett. 363, fnw204 (2016).

24. Panpetch, W. et al. Additional Candida albicans administration enhances the severity of dextran sulfate solution induced colitis mouse model through leaky gut-enhanced systemic inflammation and gut-dysbiosis but attenuated by Lactobacillus rhamnosus L34. Gut Microbes 11, 465-480 (2020).

25. Panpetch, W. et al. Candida administration worsens cecal ligation and puncture-induced sepsis in obese mice through gut dysbiosis enhanced systemic inflammation, impact of pathogen-associated molecules from gut translocation and saturated fatty acid. Front. Immunol. 11, 561652. https://doi.org/10.3389/fimmu.2020.561652 (2020).

26. Shimizu, M., Hashiguchi, M., Shiga, T., Tamura, H. O. \& Mochizuki, M. Meta-analysis: Effects of probiotic supplementation on lipid profiles in normal to mildly hypercholesterolemic individuals. PLoS ONE 10, e0139795. https://doi.org/10.1371/journal.pone. 0139795 (2015).

27. Cho, Y. A. \& Kim, J. Effect of probiotics on blood lipid concentrations: A meta-analysis of randomized controlled trials. Medicine 94, e1714. https://doi.org/10.1097/MD.0000000000001714 (2015)

28. Xie, C. \& Halegoua-DeMarzio, D. Role of probiotics in non-alcoholic fatty liver disease: Does gut microbiota matter?. Nutrients 11, 2837 (2019).

29. Amiri, S., Mokarram, R. R., Khiabani, M. S., Bari, M. R. \& Khaledabad, M. A. In situ production of conjugated linoleic acid by Bifidobacterium lactis BB12 and Lactobacillus acidophilus LA5 in milk model medium. LWT 132, 109933 (2020).

30. Zhou, K. Strategies to promote abundance of Akkermansia muciniphila, an emerging probiotics in the gut, evidence from dietary intervention studies. J. Funct. Foods 33, 194-201. https://doi.org/10.1016/j.jff.2017.03.045 (2017).

31. Caputo, A. et al. Whole-genome assembly of Akkermansia muciniphila sequenced directly from human stool. Biol. Direct 10, 5. https://doi.org/10.1186/s13062-015-0041-1 (2015).

32. Pratchayasakul, W. et al. Effects of high-fat diet on insulin receptor function in rat hippocampus and the level of neuronal corticosterone. Life Sci. 88, 619-627. https://doi.org/10.1016/j.lfs.2011.02.003 (2011).

33. Sae-khow, K. et al. Pathogen-associated molecules from gut translocation enhance severity of cecal ligation and puncture sepsis in iron-overload $\beta$-thalassemia mice. J. Inflamm. Res. 13, 719-735. https://doi.org/10.2147/JIR.S273329 (2020).

34. Thim-uam, A. et al. Leaky-gut enhanced lupus progression in the Fc gamma receptor-IIb deficient and pristane-induced mouse models of lupus. Sci. Rep. 10, 777. https://doi.org/10.1038/s41598-019-57275-0 (2020).

35. Visitchanakun, P. et al. Gut leakage enhances sepsis susceptibility in iron-overloaded $\beta$-thalassemia mice through macrophage hyperinflammatory responses. Am. J. Physiol. Gastrointest. Liver Physiol. 318, 966-979. https://doi.org/10.1152/ajpgi.00337.2019 (2020).

36. Savari, F., Mard, S. A., Badavi, M., Rezaie, A. \& Gharib-Naseri, M. K. A new method to induce nonalcoholic steatohepatitis (NASH) in mice. BMC Gastroenterol. 19, 125. https://doi.org/10.1186/s12876-019-1041-x (2019).

37. Urai, T. et al. The relationship between cutaneous wounds made on obese mice or those with decreased body weight and serum leptin level. Health 8, 1015 (2016).

38. Klaikeaw, N., Wongphoom, J., Werawatganon, D., Chayanupatkul, M. \& Siriviriyakul, P. Anti-inflammatory and anti-oxidant effects of aloe vera in rats with non-alcoholic steatohepatitis. World J. Hepatol. 12, 363-377. https://doi.org/10.4254/wjh.v12.i7. $363(2020)$.

39. $\mathrm{Xu}$, Y. et al. Function of Akkermansia muciniphila in Obesity: Interactions with lipid metabolism, immune response and gut systems. Front. Microbiol. 11, 219. https://doi.org/10.3389/fmicb.2020.00219 (2020).

40. Collado, M. C., Meriluoto, J. \& Salminen, S. Measurement of aggregation properties between probiotics and pathogens: In vitro evaluation of different methods. J. Microbiol. Methods 71, 71-74. https://doi.org/10.1016/j.mimet.2007.07.005 (2007).

41. Ward, L. J. \& Timmins, M. J. Differentiation of Lactobacillus casei, Lactobacillus paracasei and Lactobacillus rhamnosus by polymerase chain reaction. Lett. Appl. Microbiol. 29, 90-92. https://doi.org/10.1046/j.1365-2672.1999.00586.x (1999).

42. Issara-Amphorn, J., Somboonna, N., Pisitkun, P., Hirankarn, N. \& Leelahavanichkul, A. Syk inhibitor attenuates inflammation in lupus mice from FcgRIIb deficiency but not in pristane induction: The influence of lupus pathogenesis on the therapeutic effect. Lupus 29, 1248-1262 (2020).

43. Schloss, P. D. et al. Introducing mothur: open-source, platform-independent, community-supported software for describing and comparing microbial communities. Appl. Environ. Microbiol. 75, 7537-7541. https://doi.org/10.1128/AEM.01541-09 (2009).

44. Bulan, D. E. et al. Spatial and seasonal variability of reef bacterial communities in the upper gulf of Thailand. Front. Mar. Sci. 5, 441. https://doi.org/10.3389/fmars.2018.00441 (2018).

45. Segata, N. et al. Metagenomic biomarker discovery and explanation. Genome Biol. 12, R60. https://doi.org/10.1186/gb-2011-12-6r60 (2011).

46. Jaroonwitchawan, T. et al. Dysregulation of lipid metabolism in macrophages is responsible for severe endotoxin tolerance in FcgRIIB-deficient lupus mice. Front. Immunol. 11, 959. https://doi.org/10.3389/fimmu.2020.00959 (2020).

47. Tseng, C. H. \& Wu, C. Y. The gut microbiome in obesity. J. Formos Med. Assoc 118(Suppl 1), S3-S9. https://doi.org/10.1016/j.jfma. 2018.07.009 (2019).

48. Goldstein, E. J., Citron, D. M., Peraino, V. A. \& Cross, S. A. Desulfovibrio desulfuricans bacteremia and review of human Desulfovibrio infections. J. Clin. Microbiol. 41, 2752-2754. https://doi.org/10.1128/jcm.41.6.2752-2754.2003 (2003).

49. Zheng, H. et al. Modulation of gut microbiome composition and function in experimental colitis treated with sulfasalazine. Front. Microbiol. 8, 1703. https://doi.org/10.3389/fmicb.2017.01703 (2017).

50. Panpetch, W. et al. Oral administration of live-or heat-killed Candida albicans worsened cecal ligation and puncture sepsis in a murine model possibly due to an increased serum (1 $\rightarrow 3)$ - $\beta$-D-glucan. PLoS ONE 12, e0181439 (2017).

51. Zhang, T., Li, Q. Q., Cheng, L., Buch, H. \& Zhang, F. Akkermansia muciniphila is a promising probiotic. Microb. Biotechnol. 12, 1109-1125. https://doi.org/10.1111/1751-7915.13410 (2019).

52. Di Rienzi, S. C. et al. The human gut and groundwater harbor non-photosynthetic bacteria belonging to a new candidate phylum sibling to Cyanobacteria. Elife 2, e01102. https://doi.org/10.7554/eLife.01102 (2013).

53. den Besten, G. et al. The role of short-chain fatty acids in the interplay between diet, gut microbiota, and host energy metabolism. J. Lipid Res. 54, 2325-2340. https://doi.org/10.1194/jlr.R036012 (2013).

54. Geerlings, S. Y., Kostopoulos, I., de Vos, W. M. \& Belzer, C. Akkermansia muciniphila in the human gastrointestinal tract: When, where, and how?. Microorganisms 6, 75. https://doi.org/10.3390/microorganisms6030075 (2018).

55. VanWagner, L. B. \& Rinella, M. E. Extrahepatic manifestations of nonalcoholic fatty liver disease. Curr. Hepatol. Rep. 15, 75-85. https://doi.org/10.1007/s11901-016-0295-9 (2016).

56. Ellulu, M. S., Patimah, I., Khazaai, H., Rahmat, A. \& Abed, Y. Obesity and inflammation: the linking mechanism and the complications. Arch. Med. Sci. 13, 851-863. https://doi.org/10.5114/aoms.2016.58928 (2017). 
57. Jurgonski, A., Juskiewicz, J. \& Zdunczyk, Z. A high-fat diet differentially affects the gut metabolism and blood lipids of rats depending on the type of dietary fat and carbohydrate. Nutrients 6, 616-626. https://doi.org/10.3390/nu6020616 (2014).

58. Chen, L., Li, H., Li, J., Chen, Y. \& Yang, Y. Lactobacillus rhamnosus GG treatment improves intestinal permeability and modulates microbiota dysbiosis in an experimental model of sepsis. Int. J. Mol. Med. 43, 1139-1148. https://doi.org/10.3892/ijmm.2019.4050 (2019).

59. Shin, N. R., Whon, T. W. \& Bae, J. W. Proteobacteria: Microbial signature of dysbiosis in gut microbiota. Trends Biotechnol. 33, 496-503. https://doi.org/10.1016/j.tibtech.2015.06.011 (2015).

60. Alauzet, C. \& Jumas-Bilak, E. The Phylum Deferribacteres and the Genus Caldithrix. The Prokaryotes (Springer, Berlin, 2014).

61. Bloom, S. M. et al. Commensal Bacteroides species induce colitis in host-genotype-specific fashion in a mouse model of inflammatory bowel disease. Cell Host Microbe 9, 390-403. https://doi.org/10.1016/j.chom.2011.04.009 (2011).

62. Boutagy, N. E., McMillan, R. P., Frisard, M. I. \& Hulver, M. W. Metabolic endotoxemia with obesity: Is it real and is it relevant?. Biochimie 124, 11-20. https://doi.org/10.1016/j.biochi.2015.06.020 (2016).

63. Zhang, C. et al. Structural resilience of the gut microbiota in adult mice under high-fat dietary perturbations. ISME J. 6, 1848-1857. https://doi.org/10.1038/ismej.2012.27 (2012).

64. Hildebrandt, M. A. et al. High-fat diet determines the composition of the murine gut microbiome independently of obesity. Gastroenterology 137(1716-1724), e1711-1712. https://doi.org/10.1053/j.gastro.2009.08.042 (2009).

65. Kobyliak, N. et al. Probiotics in prevention and treatment of obesity: a critical view. Nutr. Metab. (Lond) 13, 14. https://doi.org/ 10.1186/s12986-016-0067-0 (2016)

66. Liguori, R. et al. Selection of the strain Lactobacillus acidophilus ATCC 43121 and its application to brewers' spent grain conversion into lactic acid. Biomed. Res. Int. https://doi.org/10.1155/2015/240231 (2015).

67. Monteagudo-Mera, A., Rastall, R. A., Gibson, G. R., Charalampopoulos, D. \& Chatzifragkou, A. Adhesion mechanisms mediated by probiotics and prebiotics and their potential impact on human health. Appl. Microbiol. Biotechnol. 103, 6463-6472. https://doi. org/10.1007/s00253-019-09978-7 (2019).

68. Rinninella, E. et al. What is the healthy gut microbiota composition? A changing ecosystem across age, environment, diet, and diseases. Microorganisms 7, 14 (2019).

69. Naito, Y., Uchiyama, K. \& Takagi, T. A next-generation beneficial microbe: Akkermansia muciniphila. J. Clin. Biochem. Nutr. 63, 33-35. https://doi.org/10.3164/jcbn.18-57 (2018).

70. Everard, A. et al. Cross-talk between Akkermansia muciniphila and intestinal epithelium controls diet-induced obesity. Proc. Natl. Acad. Sci. USA 110, 9066-9071. https://doi.org/10.1073/pnas.1219451110 (2013).

71. Li, J., Lin, S. Q., Vanhoutte, P. M., Woo, C. W. \& Xu, A. M. Akkermansia muciniphila protects against atherosclerosis by preventing metabolic endotoxemia-induced inflammation in apoe(-/-) Mice. Circulation https://doi.org/10.1161/Circulationaha.115.019645 (2016).

\section{Author contributions}

T.O. conceived of the idea, collected data, analyzed data, drafted the manuscript; K.P. conceived of the idea, edited the manuscript, manage project, secure funding; P.V. performed in vivo study; W.S. performed gut leakage measurement; S.K. prepared probiotics, performed in vivo and in vitro study, edited the manuscript; LW performed microbiome analysis; C.K. performed microbiome analysis; N.N. performed microbiome analysis; S.S. provided probiotics; N.S. performed microbiome analysis; A.L. conceived of the idea, collected data, analyzed data, drafted the manuscript; All authors reviewed the manuscript.

\section{Funding}

This research was supported by Thailand Research Fund (RDG6150124), Thailand Government Fund (RSA6080023), the Program Management Unit for Human Resources \& Institutional Development Research and Innovation-CU [Global Partnership B16F630071 and Flagship B05F630073], and Matching fund (RA-MF12/62). TO was funded by Second Century Fund (C2F), Chulalongkorn University.

\section{Competing interests}

The authors declare no competing interests.

\section{Additional information}

Correspondence and requests for materials should be addressed to K.P. or A.L.

Reprints and permissions information is available at www.nature.com/reprints.

Publisher's note Springer Nature remains neutral with regard to jurisdictional claims in published maps and institutional affiliations.

(c) (i) Open Access This article is licensed under a Creative Commons Attribution 4.0 International cc) License, which permits use, sharing, adaptation, distribution and reproduction in any medium or format, as long as you give appropriate credit to the original author(s) and the source, provide a link to the Creative Commons licence, and indicate if changes were made. The images or other third party material in this article are included in the article's Creative Commons licence, unless indicated otherwise in a credit line to the material. If material is not included in the article's Creative Commons licence and your intended use is not permitted by statutory regulation or exceeds the permitted use, you will need to obtain permission directly from the copyright holder. To view a copy of this licence, visit http://creativecommons.org/licenses/by/4.0/.

(C) The Author(s) 2021 